\title{
The Efficacy of Questionnaire-based Evaluation in Determining the Incidence of Recent Pseudophakic Dysphotopsia
}

\author{
Szilvia Pál', Andreea Dana Fișuș1,2, Florina Vultur¹,2, Karin Horvath,2 \\ ${ }^{1}$ University of Medicine and Pharmacy, Tîrgu Mures, Romania \\ 2 Ophtalmology Clinic, Mureș County Hospital, Tîrgu Mureș, Romania
}

\section{CORRESPONDENCE}

\section{Szilvia Pál}

Str. Nicolae Grigorescu nr. 17

540096 Tîrgu Mureș, Romania

Tel: +40 754654951

E-mail: pal.szilvia.93@gmail.com

\section{ARTICLE HISTORY}

Received: January 28, 2018

Accepted: February 22, 2018
Andreea Dana Fișus • Str. Gheorghe Marinescu nr. 38 , 540139 Tîrgu Mureș, Romania. Tel:+40 265215551 Florina Vultur • Str. Gheorghe Marinescu nr. 38 , 540139 Tîrgu Mureș, Romania. Tel:+40 265215551

Karin Horvath • Str. Gheorghe Marinescu nr. 38 540139 Tîrgu Mures, Romania. Tel:+40 265215551

\begin{abstract}
Background: Dysphotopsias are optical side effects experienced by patients who underwent cataract surgery. This unwanted photic phenomenon has gained ground and is a major postoperative concern. Visual acuity is not sufficient in evaluating the postoperative visual function. The aim of this study was to determine the efficacy of using a preexistent questionnaire in determining the presence of dysphotopsia. Material and method: We conducted a prospective study, using the modified Visual Function Index (VF-14) and the Ocular Surface Disease Index (OSDI) surveys, completed on patients that underwent uncomplicated phacoemulsification with intraocular lens implantation between November 2016 and November 2017. Patients included in the study had no known ocular comorbidities and had no other possible postoperative explanation for these visual phenomena. Three weeks after the surgery, the questionnaire was filled up by one individual examiner. Results: Of the 50 patients considered, 37 patients met all the inclusion criteria and were successfully enrolled in the study, with a mean age of 75.88 years. Dysphotopsia phenomena were present in $13.51 \%$ of cases; $60 \%$ of these patients described the presence of positive dysphotopsia, and $40 \%$ complained of temporal shadows. The best corrected visual acuity was over 0.8 in $75.67 \%$ of the cases. Conclusion: Although there is no objective test to diagnose this early postoperative complication, pseudophakic dysphotopsia should not be overlooked and additional chair time is needed.
\end{abstract}

Keywords: questionnaire, dysphotopsia, cataract, intraocular lens, temporal shadow

\section{INTRODUCTION}

Cataract surgery with intraocular lens (IOL) implantation is currently the most commonly performed ophthalmic surgery. ${ }^{1}$

As a result of increasing life expectancy, the number of people with age-related eye diseases and consecutive visual deterioration will raise significantly in the near future ${ }^{2}$ cataract being the first leading cause of blindness. ${ }^{3}$

The aim of contemporary cataract surgery is more than removing the opacified lens; it also pursues to restore visual function at diverse distances. ${ }^{4}$ Even in case of an uneventful cataract surgery, in which the results of the intervention 
would support a higher satisfaction, patients often complain of seeing unwanted images.

This phenomenon was first described by Tester $e$ t al. as "dysphotopsia" to illustrate any light-related visual complaints encountered in phakic and pseudophakic patients. ${ }^{5}$ These unwanted optical images can be divided into two types, positive and negative. ${ }^{6}$ Positive dysphotopsias are most often described as flashes, arcs, halos, or sprinkles, bright artifacts. Contrarily, negative dysphotopsias block light from reaching the retina, resulting in shadows or dark spots that usually appear in the temporal field. $., 7,8$

As mentioned, dysphotopsias decrease the patients' postoperative visual outcome and satisfaction, and in cases with permanent, severe symptoms it can lead to IOL exchange. 5,9

Fortunately, this symptom is transient for most patients and only close observation is needed. If the symptom does not disappear in 3 to 4 months or later, further procedures may be necessary. Possible solutions include the following:

- surgical: YAG laser capsulectomy, a secondary piggyback lens, reverse optic capture, IOL exchange (in severe cases) $)^{10,11}$;

- non-surgical: the use of thick-frame glasses, pupil dilatation in the case of negative dysphotopsia. ${ }^{11}$

The aim of this study was to determine the efficacy of using a preexistent questionnaire in determining the presence of dysphotopsia.

\section{MATERIAL AND METHODS}

We conducted a questionnaire-based prospective study that included patients who underwent uneventful phacoemulsification and IOL implantation for senile cataract at the Clinic of Ophthalmology in Tîrgu Mureș.

Surgeries were performed by the same doctor. The surgical technique was identical in all cases and included topical anesthesia, a clear-corneal incision (a $2.2 \mathrm{~mm}$ main incision and two $1.2 \mathrm{~mm}$ side incisions), a curvilinear capsulorhexis (5-5.5 mm), and in-the-bag implantation of an aspheric monofocal lens with square edges, with an optic size of $6.0 \mathrm{~mm}$ and a $13.0 \mathrm{~mm}$ overall diameter.

Three weeks after the operation, the surgeon performed the ocular follow-up by analyzing the visual acuity, autorefraction, and the lens clarity with the sit lamp, thus excluding any objective cause of possible visual symptoms.

The questionnaire was performed by one individual examiner. Each evaluation was restricted to one eye per patient. A total of 13 questions were used from the modified
Visual Function Index (VF-14, introduced in 1996) and Ocular Surface Disease Index (OSDI, introduced in 1997) between November 2016 and November 2017, evaluating patient satisfaction and ocular complaints. The patients' charts included the following data: age, gender, ocular condition and visual acuity before cataract surgery and during follow-up, the best corrected vision, the ocular refraction, as well as the type and power of the implanted IOL. All the patients offered a verbal informed consent for participation in the study.

The included patients were 65 years of age or older, had no known ocular comorbidities and no possible postoperative explanation for any unwanted visual phenomena. The exclusion criteria comprised: mental or physical impairment; corneal, macular, or retinal pathology that affected the vision; glaucoma; dry-eye syndrome, or any cause of central visual acuity loss, including dysfunction of the optic nerve. Postoperative complications likely to affect visual quality, such as eye inflammation, corneal or macular edema, intraocular lens dislocation, refractive errors (of more than \pm 1.50 spherical/cylinder diopter) also disqualified subjects from enrollment in the study.

\section{RESULTS}

Of the 50 patients considered, 37 patients met all the inclusion criteria and were successfully enrolled in the study, with a mean age of 75.88 years (range: $65-89$ years), including 20 (54\%) women. Postoperative best corrected vision above 0.80 was achieved in $75.67 \%$ of the cases (Figure 1).

Patients were asked to rank their satisfaction from 1 to 5 ( 1 being unsatisfied and 5 very satisfied); $94.59 \%$ of them were very content. None of them was unhappy with the surgery results.

In the study, $43.24 \%$ were within the range of \pm 0.50 DSph refraction, and $81.08 \%$ within \pm 1.00 DSph refraction.

At the three-week follow-up, $13.51 \%(\mathrm{n}=5)$ of patients related the presence of dysphotopsias, $60 \%(n=3)$ of them experienced unwanted optical images, and $40 \%(n=2)$ dark spots in the temporal visual field (Figure 2). These dysphotopsias disappeared after two weeks, only one patient complained of permanent symptoms.

\section{DISCUSSIONS}

The purpose of the first nine questions from the National Eye Institute Visual Functioning Questionnaire was to find out the overall postoperative satisfaction of the patients, 


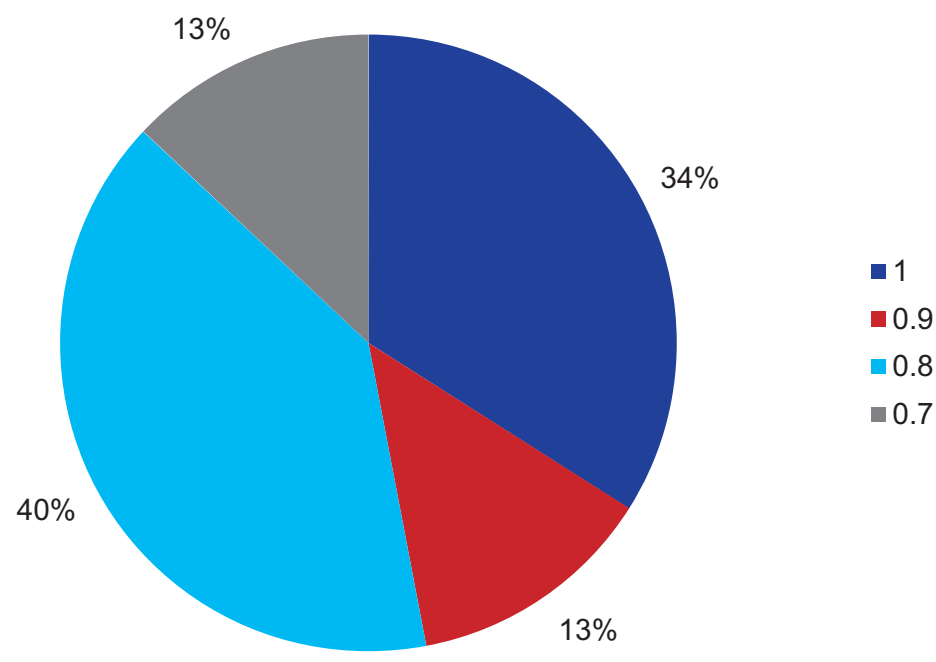

FIGURE 1. Postoperative best corrected vision

while the last four questions were used to detect a dry-eye syndrome, which can also cause refractive issues and disqualify patients from participation.

Not all queries reached statistical significance. Asking whether they had visual difficulties during day- or nighttime driving was not applicable to all patients. Many of them either did not drive at all due to their age, or they have given up driving in those three weeks after cataract surgery. However, we considered this query useful, because while driving, especially at night, unwanted light phenomena (halo) may occur.
Even when dysphotopsias occurred, patients were satisfied with the results of the surgery. One of the reasons may be that the vision (and with this, the quality of their lives) was lower, thus the increase of vision doubtlessly increased their satisfaction (Figure 3).12 In the literature, the incidence of dark temporal shadows ranges between $0.1 \%$ and $6.7 \%$ of eyes implanted with different monofocal IOLs. ${ }^{13}$

According to current studies, there are more patients suffering from these visual symptoms, primarily because it is not a routine question asked during the follow-up visits. ${ }^{14}$

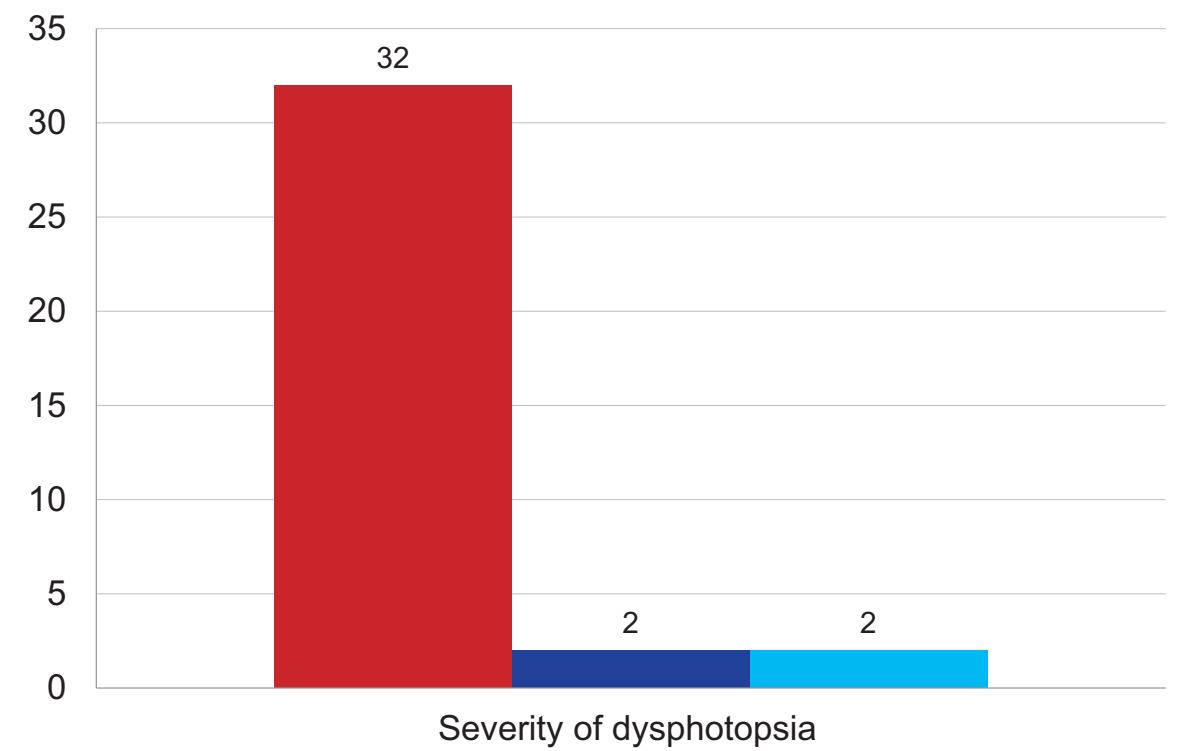

FIGURE 2. Severity of dysphotopsia 


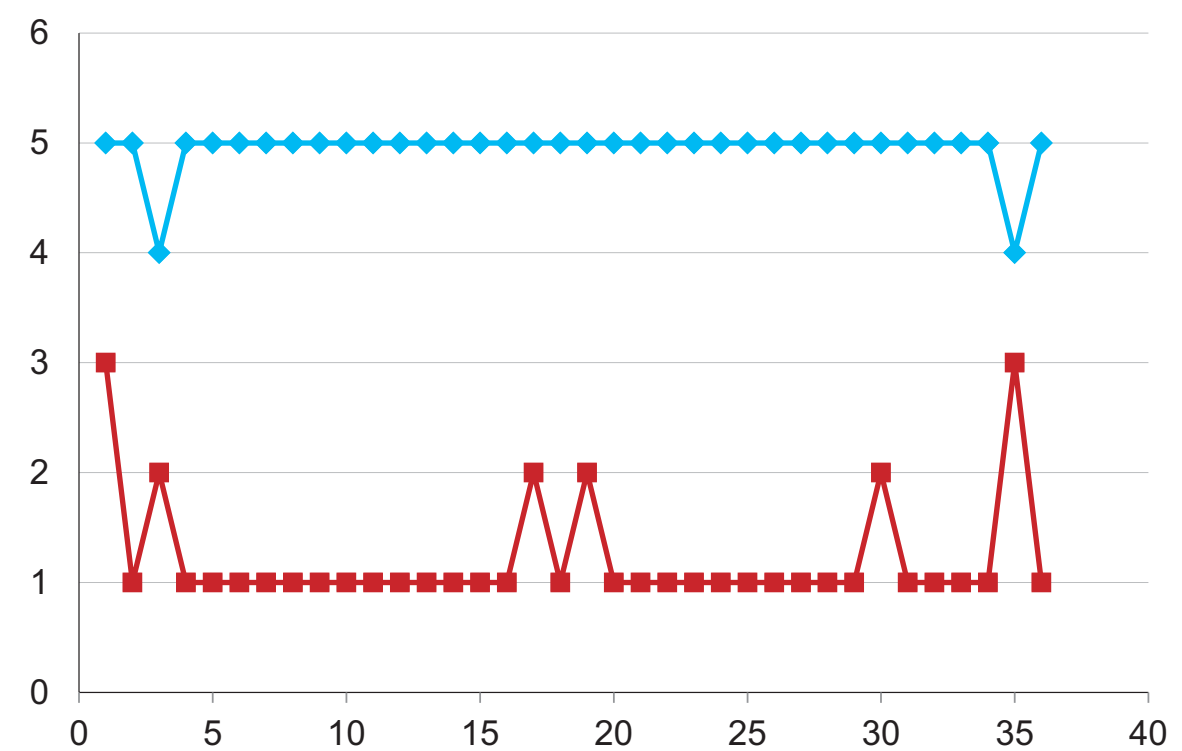

FIGURE 3. The correlation between visual complaints and postoperative satisfaction

Although women live longer than men and thus are more likely to develop cataract (they also have a slightly higher risk to develop age-related ocular diseases and have a reluctance in accepting eye-care services ${ }^{3}$ ), there was no statistically significant difference between female and male participants in this study. On the contrary, Lewallen et al. determined that in Asia and Africa, women do not receive cataract surgery at the same rate as men. ${ }^{3,15}$

Even though younger patients with larger scotopic pupils might be more exposed to dysphotopsia, and they are generally more critical about their vision compared with older pseudophakic patients, ${ }^{7,16}$ the majority of dysphotopsias occurred at patients aged above 80 years.

The patients included in our study received hydrophobic acrylic IOLs, due to significantly lower rates of posterior capsular opacification (PCO), in comparison with hydrophilic acrylic IOLs. The square-edge lens design shows low incidence of lens epithelial cell migration, but a higher incidence of dysphotopsias due to internal reflections at the rectangular edge of the IOL. ${ }^{16-19}$ As reported in a 2014 article, "rounded-edge IOLs would reduce the incidence, but it is not worth the increased rate of PCO”. ${ }^{14}$

\section{LIMITATIONS OF THE STUDY}

We consider that, to some degree, answers might have been induced by the questionnaire, as many of the patients did not report the symptoms in question prior to the examination.
Despite some degree of bias, the questionnaire-based evaluation is the only method of evaluating the presence of dysphotopsia.

The weakness of this study is the small number of participants, which may be explained by the rigor of the eligibility requirements for enrollment.

\section{CONCLUSION}

Although there is no objective test to diagnose this postoperative light-related phenomenon, evaluation of the visual acuity is not sufficient in the assessment of postoperative visual function.

Taking into consideration the frequency of dysphotopsias that were detected in the examined patients, we can conclude that preexistent questionnaires demonstrate good reliability and provide an efficient way to identify dysphotopsias. According to our results, this survey should be part of the routine postoperative follow-up, because focusing not only on surgical results, but on postoperative contentment too, cataract surgeries could bring a higher satisfaction and treatment options could bring more specific results.

\section{CONFLICT OF INTEREST}

Nothing to declare.

\section{REFERENCES}

1. Hu J, Sella R, Afshari NA. Dysphotopsia: a multifaceted optic phenomenon Current Opinion in Ophthalmology. 2018;29:61-68. 
2. Laitinen A, Laatikainen L, Härkänen T, Koskinen S, Reunanen A, Aromaa A. Prevalence of major eye diseases and causes of visual impairment in the adult Finnish population: a nationwide population-based survey. Acta Ophthalmologica. 2010;88:463-471.

3. Lewallen S, Courtright P. Increasing uptake of eye services by women. Community Eye Health. 2006;19:59-60.

4. Lubiński W, Gronkowska-Serafin J, Podborączyńska-Jodko K. Clinical outcomes after cataract surgery with implantation of the Tecnis ZMBOO multifocal intraocular lens. Medical Science Monitor. 2014;15;20:12201226.

5. Tester R, Pace NL, Samore M, Olson RJ. Dysphotopsia in phakic and pseudophakic patients: Incidence and relation to commonly used intraocular lenses. Journal of Cataract \& Refractive Surgery. 2000;26:810816.

6. Holladay JT, Lang A, Portney V. Analysis of edge glare phenomena in intraocular lens edge designs. Journal of Cataract \& Refractive Surgery. 1999;25:748-752.

7. Davison JA. Positive and negative dysphotopsia in patients with acrylic intraocular lenses. Journal of Cataract \& Refractive Surgery. 2000;26:1346-1355.

8. Schwiegerling J. Recent developments in pseudophakic dysphotopsia. Current Opinion in Ophthalmology. 2006;17:27-30.

9. Vámosi P, Csákány B, Németh J. Intraocular lens exchange in patients with negative dysphotopsia symptoms. Journal of Cataract \& Refractive Surgery. 2010;36:418-424.

10. Michelson MA, Holladay JT. The Intersection of Optics and NeuroOphthalmology: The Enigma of Pseudophakic Dysphotopsia. Journal of Neuro-Ophthalmology. 2015;35:109-111.
11. Caceres V. Treating, eliminating negative dysphotopsia. Eye World. 2014;19:38-40.

12. Fişuş AD, Madaras Z, Horváth KU. The Prevalence of Dysphotopsia in Patients with Recent Cataract Surgery. Acta Medica Marisiensis. 2017;63:14.

13. Makhotkina NY, Nijkamp MD, Berendschot TTJM, van den Borne B, Nuijts RMMA. Effect of active evaluation on the detection of negative dysphotopsia after sequential cataract surgery: discrepancy between incidences of unsolicited and solicited complaints. Acta Ophthalmologica. 2018;96:81-87.

14. Dalton M. Pseudophakic dysphotopsia - Understanding positive dysphotopsia. EyeWorld Online. https://www.eyeworld.org/articleunderstanding-positive-dysphotopsia (April 2014)

15. Lewallen S, Mousa A, Bassett K, Courtright P. Cataract surgical coverage remains lower in females. British Journal of Ophthalmology. 2009;93:295298.

16. Li Y, Wang J, Chen Z, Tang $X$. Effect of Hydrophobic Acrylic versus Hydrophilic Acrylic Intraocular Lens on Posterior Capsule Opacification: Meta-Analysis. Bruns H, ed. PLoS ONE. 2013;8:e77864.

17. Leaming DV. Practice styles and preferences of ASCRS members - 2003 survey. Journal of Cataract \& Refractive Surgery. 2004;30:892-900.

18. Hazra S, Palui H, Vemuganti GK. Comparison of design of intraocular lens versus the material for PCO prevention. International Journal of Ophthalmology. 2012;5:59-63.

19. Findl O. Intraocular lens materials and design. In: Colvard DM, ed. Achieving Excellence in Cataract Surgery: A Step-by-Step Approach. Los Angeles, CA: Colvard DM, 2009; p. 96. 